\title{
A hybrid mean value involving a new sum and Kloosterman sums
}

Xiaohan Wang ${ }^{1}$ and Xiaoxue $\mathrm{Li}^{\mathrm{i}^{*}}$

*Correspondence: Ixx20072012@163.com

2Department of Mathematics, Northwest University, Xi'an, Shaanxi, P.R. China

Full list of author information is available at the end of the article

\section{Abstract}

In this paper, we introduce a new sum, analogous to Cochrane sums, and use elementary and analytic methods to study the hybrid mean value problem involving this sum and Kloosterman sums, and we give an interesting asymptotic formula for it. MSC: Primary 11L40; 11F20

Keywords: new sum analogous to Cochrane sums; Kloosterman sums; hybrid mean value; asymptotic formula

\section{Introduction}

Let $q$ be a natural number and $h$ an integer with $(h, q)=1$. The Cochrane sum $C(h, q)$ is defined by

$$
C(h, q)=\sum_{a=1}^{q}\left(\left(\frac{\bar{a}}{q}\right)\right)\left(\left(\frac{a h}{q}\right)\right),
$$

where

$$
((x))= \begin{cases}x-[x]-\frac{1}{2}, & \text { if } x \text { is not an integer } \\ 0, & \text { if } x \text { is an integer }\end{cases}
$$

$\bar{a}$ is defined by $a \bar{a} \equiv 1 \bmod q$, and $\sum_{a=1}^{\prime q}$ denotes the summation over all $1 \leq a \leq q$ such that $(a, q)=1$.

This sum was introduced by Professor Todd Cochrane, and it has been studied by many authors, and one obtained many interesting results. Related works can be found in [1-3] and [4-11]. For example, Zhang [11] studied the hybrid mean value properties of Cochrane sums and Kloosterman sums and proved that for any prime $p>3$, we have the asymptotic formula

$$
\sum_{h=1}^{p-1} K(h, 1 ; p) C(h, p)=\frac{-1}{2 \pi^{2}} p^{2}+O\left(p \cdot \exp \left(\frac{3 \ln p}{\ln \ln p}\right)\right),
$$

where $\exp (y)=e^{y}$,

$$
K(m, n ; q)=\sum_{a=1}^{q} e\left(\frac{m a+n \bar{a}}{q}\right)
$$

denotes the Kloosterman sum, and $e(y)=e^{2 \pi i y}$. 
Now for any odd prime $p>1$ and integer $h$ with $(h, q)=1$, we define another sum analogous to the Cochrane sum as follows:

$$
C_{1}(h, p)=\sum_{a=1}^{\frac{p-1}{2}}\left(\left(\frac{\bar{a}^{2}}{p}\right)\right)\left(\left(\frac{a^{2} h}{p}\right)\right) .
$$

The main purpose of this paper is using elementary and analytic methods to study the hybrid mean value problem involving $C_{1}(h, p)$ and the Kloosterman sum and give an asymptotic formula for it. That is, we shall prove the following.

Theorem Let $p$ be an odd prime. Then we have the asymptotic formula

$$
\sum_{h=1}^{p-1} K(h, 1 ; p) C_{1}(h, p)=\frac{-1}{4 \pi^{2}} \cdot p^{2}+O\left(p^{3 / 2} \cdot \exp \left(\frac{3 \ln p}{\ln \ln p}\right)\right) .
$$

For general odd number $q>2$, whether there exists an asymptotic formula for

$$
\sum_{h=1}^{q} K(h, 1 ; q) C_{1}(h, q)
$$

is still an open problem.

\section{Several lemmas}

In this section, we shall give several lemmas, which are necessary in the proof of our theorem. Hereinafter, we shall use many properties of Gauss sums; all of these can be found in reference [12], so they will not be repeated here. First we have the following.

Lemma 1 Let $p$ be an odd prime. Then for any integer a with $(a, p)=1$, we have the identity

$$
C(a, p)=\frac{-1}{\pi^{2}(p-1)} \sum_{\substack{\chi \bmod p \\ \chi(-1)=-1}} \bar{\chi}(a) \tau^{2}(\chi) L^{2}(1, \bar{\chi}),
$$

where $\chi$ runs through the Dirichlet characters $\bmod p$ with $\chi(-1)=-1$, and $\tau(\chi)=$ $\sum_{a=1}^{p-1} \chi(a) e\left(\frac{a}{p}\right)$ denotes the classical Gauss sum corresponding to $\chi$.

Proof See reference [11].

Lemma 2 Let $p$ be an odd prime and $h$ an integer with $(h, p)=1$. We define $C_{2}(h, p)$ as follows:

$$
C_{2}(h, p)=\sum_{a=1}^{p-1}\left(\frac{a}{p}\right)\left(\left(\frac{\bar{a}}{p}\right)\right)\left(\left(\frac{a h}{p}\right)\right) .
$$

Then we have the identity

$$
C_{2}(h, p)= \begin{cases}-\frac{1}{\pi^{2}} \frac{1}{p-1} \sum_{\chi \bmod p} \bar{\chi}(h) \tau(\chi) \tau\left(\chi \chi_{2}\right) L(1, \bar{\chi}) L\left(1, \bar{\chi} \chi_{2}\right), & \text { if } p \equiv 1 \bmod 4 \\ 0, & \text { if } p \equiv 3 \bmod 4,\end{cases}
$$

where $\chi_{2}=\left(\frac{*}{p}\right)$ denotes the Legendre symbol. 
Proof From the orthogonality relation for characters $\bmod p$ we have

$$
\begin{aligned}
C_{2}(h, p) & =\frac{1}{p-1} \sum_{a=1}^{p-1} \sum_{b=1}^{p-1}\left(\frac{a}{p}\right) \sum_{\chi \bmod p} \chi(a b)\left(\left(\frac{a}{p}\right)\right)\left(\left(\frac{h b}{p}\right)\right) \\
& =\frac{1}{p-1} \sum_{\chi \bmod p}\left(\sum_{a=1}^{p-1}\left(\frac{a}{p}\right) \chi(a)\left(\left(\frac{a}{p}\right)\right)\right)\left(\sum_{b=1}^{p-1} \chi(b)\left(\left(\frac{h b}{p}\right)\right)\right) .
\end{aligned}
$$

If $\chi=\chi_{0}$ is the principal character $\bmod p$, then we have

$$
\sum_{b=1}^{p-1} \chi(b)\left(\left(\frac{h b}{p}\right)\right)=\sum_{b=1}^{p-1}\left(\left(\frac{b}{p}\right)\right)=\sum_{b=1}^{p-1}\left(\frac{b}{p}-\frac{1}{2}\right)=0 .
$$

If $\chi$ is an even character $\bmod p$ (that is, $\chi$ is a non-principal character and $\chi(-1)=1$ ), then we have

$$
\begin{aligned}
\sum_{b=1}^{p-1} \chi(b)\left(\left(\frac{h b}{p}\right)\right) & =\bar{\chi}(h) \sum_{b=1}^{p-1} \chi(b)\left(\frac{b}{p}-\frac{1}{2}\right) \\
& =\bar{\chi}(h) \frac{1}{p}\left(\sum_{b=1}^{p-1} \chi(b) b-\frac{1}{2} \sum_{b=1}^{p-1} \chi(b)\right)=\bar{\chi}(h) \frac{1}{p} \sum_{b=1}^{p-1} \chi(b) b .
\end{aligned}
$$

Since

$$
\begin{aligned}
\sum_{b=1}^{p-1} \chi(b) b & =\sum_{b=1}^{p-1} \chi(p-b)(p-b)=\sum_{b=1}^{p-1} \chi(b)(p-b) \\
& =p \sum_{b=1}^{p-1} \chi(b)-\sum_{b=1}^{p-1} \chi(b) b=-\sum_{b=1}^{p-1} \chi(b) b
\end{aligned}
$$

so that

$$
\sum_{b=1}^{p-1} \chi(b) b=0
$$

From this identity, (2), and (3) we know that if $\chi$ is an even character $\bmod p$, then

$$
\sum_{b=1}^{p-1} \chi(b)\left(\left(\frac{h b}{p}\right)\right)=0 .
$$

If $\chi$ is an odd character $\bmod p$ and $p \equiv 3 \bmod 4$. Let $\chi_{2}=\left(\frac{*}{p}\right)$ denote the Legendre symbol, then $\chi \chi_{2}$ must be an even character $\bmod p$, so we have

$$
\sum_{a=1}^{p-1}\left(\frac{a}{p}\right) \chi(a)\left(\left(\frac{a}{p}\right)\right)=0 .
$$


If $\chi$ is an odd character $\bmod p$ and $p \equiv 1 \bmod 4$, then $\chi \chi_{2}$ must be an odd character $\bmod p$, so we have

$$
\begin{aligned}
\sum_{a=1}^{p-1}\left(\frac{a}{p}\right) \chi(a)\left(\left(\frac{a}{p}\right)\right) & =\frac{1}{p} \sum_{a=1}^{p-1}\left(\frac{a}{p}\right) \chi(a) a \\
& =\frac{i}{\pi} \tau\left(\chi \chi_{2}\right) L\left(1, \bar{\chi} \chi_{2}\right)
\end{aligned}
$$

and

$$
\sum_{b=1}^{p-1} \chi(b)\left(\left(\frac{h b}{p}\right)\right)=\frac{\bar{\chi}(h)}{p} \sum_{b=1}^{p-1} \chi(b) b=\frac{i}{\pi} \bar{\chi}(h) \tau(\chi) L(1, \bar{\chi}) .
$$

Combining (1), (2), (4)-(7) we may immediately deduce the identity

$$
\begin{aligned}
C_{2}(h, p) & =\frac{1}{p-1} \sum_{a=1}^{p-1} \sum_{b=1}^{p-1}\left(\frac{a}{p}\right) \sum_{\chi \bmod p} \chi(a b)\left(\left(\frac{a}{p}\right)\right)\left(\left(\frac{h b}{p}\right)\right) \\
& =\frac{1}{p-1} \sum_{\chi \bmod p}\left(\sum_{a=1}^{p-1}\left(\frac{a}{p}\right) \chi(a)\left(\left(\frac{a}{p}\right)\right)\right)\left(\sum_{b=1}^{p-1} \chi(b)\left(\left(\frac{h b}{p}\right)\right)\right) \\
& = \begin{cases}-\frac{1}{\pi^{2}} \frac{1}{p-1} \sum_{\chi \bmod p} \bar{\chi}(h) \tau(\chi) \tau\left(\chi \chi_{2}\right) L(1, \bar{\chi}) L\left(1, \bar{\chi} \chi_{2}\right), & \text { if } p \equiv 1 \bmod 4 ; \\
0, & \text { if } p \equiv 3 \bmod 4 .\end{cases}
\end{aligned}
$$

This proves Lemma 2.

Lemma 3 Let $p>3$ be an odd prime, then we have the asymptotic formula

$$
\sum_{\substack{\chi \bmod p \\ \chi(-1)=-1}} L^{2}(1, \chi)=\frac{1}{2} p+O\left(\exp \left(\frac{3 \ln p}{\ln \ln p}\right)\right) .
$$

Proof For any non-principal character $\chi \bmod p$, applying Abel's identity (see Theorem 4.2 of [12]) we have

$$
L^{2}(1, \chi)=\sum_{n=1}^{p^{3}} \frac{\chi(n) d(n)}{n}+\int_{p^{3}}^{\infty} \frac{A(y, \chi)}{y^{2}} d y
$$

where $d(n)$ denotes the divisor function, and $A(y, \chi)=\sum_{p^{3}<n \leq y} \chi(n) d(n)$.

From [9] we know that for any real number $y>p^{3}$, we have the estimate

$$
\sum_{\substack{\chi \bmod p \\ \chi(-1)=-1}}|A(y, \chi)|^{2} \ll y \phi^{2}(p)
$$


From (8) and (9) we can deduce that

$$
\begin{aligned}
\sum_{\substack{\chi \bmod p \\
\chi(-1)=-1}} L^{2}(1, \chi) & =\frac{1}{2} \sum_{n=1}^{p^{3}} \frac{d(n)}{n} \sum_{\substack{\chi \bmod p \\
\chi(-1)=-1}} \chi(n)+O\left(\sum_{\substack{\chi \bmod p \\
\chi(-1)=-1}} \int_{p^{3}}^{\infty} \frac{|A(\chi, y)|}{y^{2}} d y\right) \\
& =\frac{p-1}{2} \sum_{\substack{n=1 \\
n \equiv 1 \bmod p}}^{p^{3}} \frac{d(n)}{n}-\frac{p-1}{2} \sum_{\substack{n=1 \\
n \equiv-1 \bmod p}}^{p^{3}} \frac{d(n)}{n}+O(1) \\
& =\frac{1}{2} p+O\left(\sum_{l=1}^{p^{2}} \frac{d(p l \pm 1)}{l}\right)=\frac{1}{2} p+O\left(\exp \left(\frac{3 \ln p}{\ln \ln p}\right)\right),
\end{aligned}
$$

where we have used the estimate $d(n) \ll \exp \left(\frac{(1+\epsilon) \ln n}{\ln \ln n}\right), \epsilon>0$ is any fixed real number. This proves Lemma 3.

Lemma 4 Let $p>2$ be a prime with $p \equiv 1 \bmod 4$. Then we have the estimates

$$
\sum_{a=1}^{p-1}\left|\sum_{\substack{\chi \bmod p \\ \chi(-1)=-1}} \chi(a) L(1, \bar{\chi}) L\left(1, \bar{\chi} \chi_{2}\right)\right|=O\left(p \cdot \exp \left(\frac{3 \ln p}{\ln \ln p}\right)\right) .
$$

Proof From the method of proving Lemma 3 we have

$$
\begin{aligned}
& \sum_{\substack{\chi \bmod p \\
\chi(-1)=-1}} \chi(a) L(1, \bar{\chi}) L\left(1, \bar{\chi} \chi_{2}\right) \\
& =\frac{1}{2} \sum_{n=1}^{p^{3}} \frac{\sum_{d \mid n} \chi_{2}(d)}{n} \sum_{\chi \bmod p}(\chi(a \bar{n})-\chi(-a \bar{n}))+O(1) \\
& =O\left(\frac{d(a)}{a} \cdot p\right)+O\left(\exp \left(\frac{3 \ln p}{\ln \ln p}\right)\right) .
\end{aligned}
$$

So applying (10) we may immediately deduce the estimate

$$
\begin{aligned}
& \sum_{a=1}^{p-1}\left|\sum_{\substack{\chi \bmod p \\
\chi(-1)=-1}} \chi(a) L(1, \bar{\chi}) L\left(1, \bar{\chi} \chi_{2}\right)\right| \\
& \quad=O\left(\sum_{a=1}^{p-1} \frac{d(a)}{a} p\right)+O\left(\sum_{a=1}^{p-1} \exp \left(\frac{3 \ln p}{\ln \ln p}\right)\right)=O\left(p \cdot \exp \left(\frac{3 \ln p}{\ln \ln p}\right)\right) .
\end{aligned}
$$

This proves Lemma 4.

\section{Proof of the theorem}

In this section, we shall use the lemmas to complete the proof of our theorem. For any prime $p>2$, note that the identities

$$
\sum_{h=1}^{p-1} \bar{\chi}(h) K(h, 1 ; p)=\sum_{b=1}^{p-1} e\left(\frac{\bar{b}}{p}\right) \sum_{h=1}^{p-1} \bar{\chi}(h) e\left(\frac{h b}{p}\right)=\tau(\bar{\chi}) \sum_{b=1}^{p} \chi(b) e\left(\frac{\bar{b}}{p}\right)=\tau^{2}(\bar{\chi})
$$


and

$$
C_{1}(h, p)=\frac{1}{2} \sum_{a=1}^{p-1}\left(1+\left(\frac{a}{p}\right)\right)\left(\left(\frac{\bar{a}}{p}\right)\right)\left(\left(\frac{a h}{p}\right)\right)=\frac{1}{2} C(h, p)+\frac{1}{2} C_{2}(h, p) .
$$

If $p \equiv 1 \bmod 4$, then from Lemma 1 , Lemma 2 , and the properties of the Gauss sum $\tau(\chi)$ we have

$$
\begin{aligned}
& \sum_{h=1}^{p-1} K(h, 1 ; p) C_{1}(h, p) \\
& =\frac{-1}{2 \pi^{2}(p-1)} \sum_{\substack{\chi \bmod p \\
\chi(-1)=-1}} \sum_{h=1}^{p-1} \bar{\chi}(h) K(h, 1 ; p) \tau^{2}(\chi) L^{2}(1, \bar{\chi}) \\
& +\frac{-1}{2 \pi^{2}(p-1)} \sum_{\substack{\chi \bmod p \\
\chi(-1)=-1}} \sum_{h=1}^{p-1} \bar{\chi}(h) K(h, 1 ; p) \tau(\chi) \tau\left(\chi \chi_{2}\right)(1, \bar{\chi})\left(1, \bar{\chi} \chi_{2}\right) \\
& =\frac{-1}{2 \pi^{2}(p-1)} \sum_{\substack{\chi \bmod p \\
\chi(-1)=-1}} \tau^{2}(\chi) \tau^{2}(\bar{\chi}) L^{2}(1, \bar{\chi}) \\
& +\frac{-1}{2 \pi^{2}(p-1)} \sum_{\substack{\chi \bmod p \\
\chi(-1)=-1}} \tau^{2}(\bar{\chi}) \tau(\chi) \tau\left(\chi \chi_{2}\right) L(1, \bar{\chi}) L\left(1, \bar{\chi} \chi_{2}\right) \\
& =\frac{p}{2 \pi^{2}(p-1)} \sum_{\substack{\chi \bmod p \\
\chi(-1)=-1}} \tau(\bar{\chi}) \tau\left(\chi \chi_{2}\right) L(1, \bar{\chi}) L\left(1, \bar{\chi} \chi_{2}\right) \\
& -\frac{p^{2}}{2 \pi^{2}(p-1)} \sum_{\substack{\chi \bmod p \\
\chi(-1)=-1}} L^{2}(1, \bar{\chi})
\end{aligned}
$$

Note that $\left|\tau\left(\chi_{2}\right)\right|=\sqrt{p}$ and that we have the identity

$$
\begin{aligned}
\tau(\bar{\chi}) \tau\left(\chi \chi_{2}\right) & =\sum_{a=1}^{p-1} \sum_{b=1}^{p-1} \bar{\chi}(a) \chi(b) \chi_{2}(b) e\left(\frac{a+b}{p}\right) \\
& =\sum_{a=1}^{p-1} \bar{\chi}(a) \sum_{b=1}^{p-1} \chi_{2}(b) e\left(\frac{a b+b}{p}\right)=\tau\left(\chi_{2}\right) \sum_{a=1}^{p-1} \bar{\chi}(a) \chi_{2}(a+1) \\
& =\tau\left(\chi_{2}\right) \sum_{a=1}^{p-1} \chi(a) \chi_{2}(\bar{a}+1),
\end{aligned}
$$

so from Lemma 4 we have

$$
\begin{aligned}
& \left|\sum_{\substack{\chi \bmod p \\
\chi(-1)=-1}} \tau(\bar{\chi}) \tau\left(\chi \chi_{2}\right) L(1, \bar{\chi}) L\left(1, \bar{\chi} \chi_{2}\right)\right| \\
& =\sqrt{p} \cdot\left|\sum_{\substack{\chi \bmod p \\
\chi(-1)=-1}} \sum_{a=1}^{p-1} \chi(a) \chi_{2}(\bar{a}+1) L(1, \bar{\chi}) L\left(1, \bar{\chi} \chi_{2}\right)\right|
\end{aligned}
$$




$$
\begin{aligned}
& \leq \sqrt{p} \cdot \sum_{a=1}^{p-1}\left|\sum_{\substack{\chi \bmod p \\
\chi(-1)=-1}} \chi(a) L(1, \bar{\chi}) L\left(1, \bar{\chi} \chi_{2}\right)\right| \\
& =O\left(p^{\frac{3}{2}} \cdot \exp \left(\frac{3 \ln p}{\ln \ln p}\right)\right) .
\end{aligned}
$$

Combining (11), (13), and Lemma 3 we can deduce the asymptotic formula

$$
\sum_{h=1}^{p-1} K(h, 1 ; p) C_{1}(h, p)=-\frac{1}{4 \pi^{2}} \cdot p^{2}+O\left(p^{\frac{3}{2}} \cdot \exp \left(\frac{3 \ln p}{\ln \ln p}\right)\right)
$$

If $p \equiv 3 \bmod 4$, then from Lemma 2 we know that $C_{1}(h, p)=\frac{1}{2} C(h, p)$, so from Lemma 3 and the method of proving (14) we also have

$$
\begin{aligned}
\sum_{h=1}^{p-1} K(h, 1 ; p) C_{1}(h, p) & =-\frac{1}{2 \pi^{2}} \frac{p^{2}}{p-1} \sum_{\substack{\chi \bmod p \\
\chi(-1)=-1}} L^{2}(1, \bar{\chi}) \\
& =-\frac{1}{4 \pi^{2}} \cdot p^{2}+O\left(p^{\frac{3}{2}} \cdot \exp \left(\frac{3 \ln p}{\ln \ln p}\right)\right) .
\end{aligned}
$$

Now the theorem follows from the asymptotic formulas (14) and (15).

\section{Competing interests}

The authors declare that they have no competing interests.

\section{Authors' contributions}

XW carried out the proofs of the lemmas, XL carried out the part of Introduction, XW and XL carried out the theorem's proof. All authors read and approved the final manuscript.

\section{Author details}

'Economy and Finance School, Xi'an International Studies University, Xi'an, Shaanxi, P.R. China. ${ }^{2}$ Department of Mathematics, Northwest University, Xi'an, Shaanxi, P.R. China.

\section{Acknowledgements}

The authors would like to thank the referees for their very helpful and detailed comments, which have significantly improved the presentation of this paper. This work is supported by the P.S.F. (2013JZ001) and N.S.F. (11371291) of P.R. China.

Received: 12 December 2013 Accepted: 10 January 2014 Published: 27 Jan 2014

\section{References}

1. Berndt, BC: Analytic Eisenstein series, theta-functions, and series relations in the spirit of Ramanujan. J. Reine Angew. Math. 303/304, 332-365 (1978)

2. Carlitz, L: The reciprocity theorem of Dedekind sums. Pac. J. Math. 3, 513-522 (1953)

3. Jia, CH: On the mean value of Dedekind sums. J. Number Theory 87, 173-188 (2001)

4. Conrey, JB, Fransen, E, Klein, R, Scott, C: Mean values of Dedekind sums. J. Number Theory 56, 214-226 (1996)

5. Gandhi, JM: On sums analogous to Dedekind sums. In: Proceedings of the Fifth Manitoba Conference on Numerical Mathematics, Winnipeg, Manitoba, 1975, pp. 647-655 (1976)

6. Rademacher, $\mathrm{H}$ : On the transformation of $\log \eta(\tau)$. J. Indian Math. Soc. 19, 25-30 (1955)

7. Rademacher, H: Dedekind Sums. Carus Mathematical Monographs. Math. Assoc. of America, Washington (1972)

8. Zhang, WP: A note on the mean square value of the Dedekind sums. Acta Math. Hung. 86, 275-289 (2000)

9. Zhang, WP: On the mean values of Dedekind sums. J. Théor. Nr. Bordx. 8, 429-442 (1996)

10. Zhang, WP: A sum analogous to Dedekind sums and its hybrid mean value formula. Acta Arith. 107, 1-8 (2003)

11. Zhang, WP: On a Cochrane sum and its hybrid mean value formula. J. Math. Anal. Appl. 267, 89-96 (2002)

12. Apostol, TM: Introduction to Analytic Number Theory. Springer, New York (1976) 